\title{
Kernresonanzen in kristallinen Flüssigkeiten und in kristallinflüssigen Lösungen. Teil I
}

\author{
Von Alfred Saupe \\ Physikalisches Institut der Universität Freiburg i. Br. \\ (Z. Naturforschg. 19 a, 161-171 [1964] ; eingegangen am 28. Oktober 1963)
}

\begin{abstract}
A theoretical study of nuclear magnetic resonances in liquid crystals and in liquid crystalline solutions is given. The average ordering of the molecules in liquid crystals, as far as it is of importance for nuclear resonance researches, can be described by a symmetrical matrix. With the help of this matrix the coupling constants of the effective average spin Hamiltosian can be expressed by the constants belonging to a fixed molecular orientation. For experimental work solutions in nematic liquid crystals are most useful. In such solutions high resolution spectra of the orientated solute molecules can be gained without any disturbance by intermolecular nuclear dipole-dipole interaction. Precise informations can be obtained on the intra-molecular dipole-dipole interaction, the anisotropy of chemical shift, and the quadrupole interaction. The matrix of order can be determined if the molecular structure is sufficiently known. In favorable cases the absolute sign of the indirect spin-spin coupling constants can also be determined.
\end{abstract}

Die vorliegende Arbeit befaßt sich mit den Kernresonanzspektren von kristallinflüssigen Phasen und von diamagnetischen Molekülen, die in kristallinen Flüssigkeiten gelöst sind. Der Mittelwert, auf den sich die innermolekulare Wechselwirkung reduziert und sein Zusammenhang mit der mittleren Molekülorientierung werden bestimmt, die Linienbreite der einzelnen Resonanzlinien wird diskutiert. Einige der Ergebnisse sind auch für die Elektronenspinresonanzen von Radikalen, die in kristallinen Flüssigkeiten gelöst sind, von Interesse; jedoch wird darauf nicht explizit eingegangen.

Für die Kernresonanzspektren von diamagnetischen Molekülen ist eine Spin-Hamilton-Funktion maßgebend, die vier ihrer physikalischen Bedeutung nach verschiedene Typen von bilinearen Vektorfunktionen enthält. Sie beschreiben die Wechselwirkung zwischen dem Magnetfeld und den magnetischen Kerndipolmomenten, die direkte und die indirekte Spin-Spin-Wechselwirkung der Kerne untereinander und die Wechselwirkung der Kernquadrupolmomente mit der Elektronenhülle. Die Wechselwirkungen werden durch die Translations- und Rotationsbewegung beeinflußt, so daß der effektive HamiltonOperator stark vom Aggregatzustand abhängt. Wir nehmen im folgenden stets ein starkes Magnetfeld an, d. h. die ZeEman-Terme sollen groß gegenüber allen übrigen Wechselwirkungstermen sein. Damit wird vermutlich der wichtigste Teil der möglichen Beobachtungen erfaßt.

Kernresonanzuntersuchungen mit hoher Auflösung werden gewöhnlich an isotropen Flüssigkeiten geringer Viskosität oder in isotropen Lösungen durch- geführt. In diesen Flüssigkeiten erfolgt die Diffusion der Moleküle so rasch, daß die zwischenmolekulare Wechselwirkung der Kernmomente auf einen praktisch verschwindenden Mittelwert reduziert wird, der durch die makroskopische Suszeptibilität erfaßt wird. Da auch die Rotationsbewegung der Moleküle sehr rasch erfolgt und jede Orientierung das gleiche statistische Gewicht hat, wird die innermolekulare Wechselwirkung ebenfalls stark reduziert. Die direkte Dipol-Dipol-Wechselwirkung und die Quadrupolwechselwirkung verschwinden im Mittel vollkommen, von der indirekten Spin-Spin-Wechselwirkung und der chemischen Verschiebung bleiben nur die isotropen Anteile übrig.

In Kristallen ist die Beweglichkeit der Moleküle gering. Es findet daher im allgemeinen auch keine Reduktion der Wechselwirkung, insbesondere auch nicht eine solche der zwischenmolekularen Wechselwirkung statt. Infolge der weitreichenden und besonders starken direkten Dipol-Dipol-Wechselwirkung sind die Resonanzlinien so vielfach aufgespalten, daß die Spektren nicht mehr aufgelöst werden können. Man beobachtet oft ein mehrere Gauß breites Absorptionsgebiet mit mehr oder weniger stark verwaschener Struktur. Es ist nur in einfachen Fällen möglich, die zwischenmolekulare Wechselwirkung abzutrennen und genaue Aussagen allein über die innermolekulare Wechselwirkung zu gewinnen. Zwischen diesen beiden Grenzfällen, nämlich der isotropen Flüssigkeit und dem Kristall, liegen die Verhältnisse in kristallinen Flüssigkeiten. Hier wird einerseits die zwischenmolekulare Wechselwirkung ebenso reduziert wie in isotropen Flüssigkeiten, also 
auf einen fast verschwindenden Mittelwert. Die Reduktion der innermolekularen Wechselwirkung ist aber immer weit geringer, weil es bevorzugte Winkellagen der Moleküle gibt, also nicht jeder Orientierung das gleiche statistische Gewicht zukommt. Es ist deshalb möglich, Aussagen zu erhalten, die weder am Festkörper noch an isotropen Flüssigkeiten gewonnen werden können.

Solche Kernresonanzuntersuchungen an kristallinen Flüssigkeiten und an kristallinflüssigen Lösungen können unmittelbar Aufschluß geben über:

1. Direkte Dipol-Dipol-Wechselwirkungen und damit über die mittleren Molekülorientierungen,

2. anisotrope chemische Verschiebungen,

3. Quadrupolwechselwirkungen.

Aus der Orientierung der Moleküle kann man Aussagen über die Struktur kristalliner Flüssigkeiten und über zwischenmolekulare Kräfte gewinnen, insbesondere auch über die Kräfte zwischen gelösten Molekülen und der Trägersubstanz. Aus der chemischen Verschiebung und aus der Quadrupolwechselwirkung ergeben sich Aussagen über die Elektronenstruktur der Moleküle, aus der Dipol-Dipol-Wechselwirkung solche über die relativen Kernabstände. Außerdem ist es in günstigsten Fällen möglich, die Vorzeichen der skalaren indirekten Spin-Spin-Kopplungen absolut zu bestimmen, die bekanntlich durch Messungen in isotropen Flüssigkeiten nur relativ festgelegt werden können.

\section{Die Haupttypen kristalliner Flüssigkeiten, der Ordnungsgrad und die Herstellung homogen orientierter Schichten}

Man unterscheidet drei Typen kristalliner Flüssigkeiten ${ }^{1}$, nämlich nematische, cholesterinische und smektische Flüssigkeiten. Abb. 1 zeigt die schematischen Strukturbilder. Homogen ausgerichtete Schichten dieser Flüssigkeiten verhalten sich optisch wie einachsige Kristalle.

In der nematischen Phase sind die Längsachsen der stabförmigen Moleküle mehr oder weniger vollkommen ausgerichtet. (Es wird nicht immer streng richtig sein, daß die Achse, mit der sich die Mole-

1 Über kristalline Flüssigkeiten: P. Chatelain, Bull. Soc. Franç. Miner. Krist. 77, 323 [1954]. - W. Kast, Angew. Chem. 67, 592 [1955]. - G. H. Brown u. W. G. Shaw, Chem. Rev. 57, 1049 [1957]. - G. W. Gray, Molecular Structure and the Properties of Liquid Crystals, Academic Press, London-New York 1962. küle parallel orientieren, mit der Längsachse zusammenfällt. Nach den bisherigen Erfahrungen gilt dies aber immer in ausreichender Näherung.) Außer der Parallelorientierung gibt es in der nematischen Phase kein weiteres Ordnungsprinzip. In der smektischen Phase findet man noch ein weiteres Ordnungsprinzip, nämlich die Anordnung der Molekülschwerpunkte in Ebenen, die senkrecht zur optischen Achse liegen.

Eng verwandt mit der nematischen Phase ist die cholesterinische Phase. Man könnte sie auch als die nematische Phase von optisch aktiven Substanzen bezeichnen. Ihre Struktur gleicht der einer verdrillten nematischen Schicht. Die homogen ausgerichtete Schicht einer cholesterinischen Flüssigkeit hat also keine über die ganze Schicht einheitliche Vorzugsrichtung mehr, nach der sich die Längsachsen orientieren, sondern es gibt nur noch Ebenen mit einheitlicher Vorzugsrichtung. Schreitet man senkrecht zu einer solchen Ebene weiter, dann dreht sich die Vorzugsrichtung kontinuierlich und gleichförmig. Eine Drehung um $180^{\circ}$ ist in manchen Fällen schon nach $0,2 \mu$ erreicht. Solche Schichten weisen deshalb eine extrem starke optische Aktivität auf. Die optische Achse steht senkrecht auf den Ebenen gleicher Orientierung.

Die Güte der Orientierung der Moleküllängsachsen kann durch den Ordnungsgrad $S$ gekennzeichnet werden, der durch folgende Beziehung definiert ist:

$$
S=\left(3\left\langle\cos ^{2} \Theta\right\rangle-1\right) / 2 .
$$

$\Theta$ bezeichnet den Winkel zwischen Moleküllängsachse und optischer Achse und $\left\langle\cos ^{2} \Theta\right\rangle$ den zeitlichen Mittelwert von $\cos ^{2} \Theta . S$ kann nach verschiedenen Methoden experimentell bestimmt werden ${ }^{2}$. Für nematische Flüssigkeiten kann $S$ auch nach einer einfachen Theorie $^{3}$ berechnet werden, sofern die Dichte in Abhängigkeit von der Temperatur bekannt ist.

Der Ordnungsgrad liegt in nematischen Flüssigkeiten im allgemeinen zwischen 0,4 und 0,7 . Seine Temperaturabhängigkeit ist verhältnismäßig stark. In smektischen Flüssigkeiten ist die Temperaturabhängigkeit geringer. Hier liegt der Ordnungsgrad

2 A. SaUpe u. W. Maier, Z. Naturforschg. 16 a, 816 [1961].

3 W. Maier u. A. Saupe, Z. Naturforschg. 14a, 882 [1959]; 15 a, 287 [1960]. 
nach den bisherigen Erfahrungen etwa zwischen 0,8 und 0,95 . Bei cholesterinischen Flüssigkeiten ist über $S$ noch nichts bekannt. Die mittlere Schwankung der Moleküle um die Vorzugsrichtung wird jedoch der in nematischen Flüssigkeiten entsprechen. $S$ liegt demnach vermutlich zwischen $-0,2$ und $-0,35$.

Um die Feinstruktur der Kernresonanzspektren wirklich voll auflösen zu können, müssen homogen orientierte Schichten der kristallinflüssigen Phasen verwendet werden. Diese lassen sich bei den nematischen Flüssigkeiten besonders leicht herstellen und erfordern keinerlei zusätzliche Maßnahmen, da die bei Kernresonanzuntersuchungen üblichen Magnetfeldstärken von 7000 bis $14000 \mathrm{Gauß}$ schon eine einheitliche Ausrichtung erzwingen, und zwar derart, daß die optische Achse parallel zum Feld steht. Die von den Küvetten ausgehende Randwirkung hat gegenüber diesen Feldstärken und bei den gewöhnlich verwendeten Probendurchmessern von mehr als $1 \mathrm{~mm}$ keinen störenden Einfluß mehr.

Es ist zu erwarten, daß cholesterinische Flüssigkeiten in dicken Schichten durch Magnetfelder von einigen $1000 \mathrm{Gau} ß$ ebenfalls orientiert werden, aber im Gegensatz zu nematischen Flüssigkeiten so, daß die optische Achse senkrecht zum Feld steht. Die Richtung der optischen Achse ist innerhalb der zum Feld senkrechten Ebene noch beliebig, man erhält also keine vollständig homogene Orientierung. Die verbleibende Inhomogenität ist aber ohne Einfluß auf die Kernresonanzspektren, weil es bei diesen nur auf den Winkel zwischen optischer Achse und Magnetfeld ankommt.

Smektische Flüssigkeiten lassen sich durch Magnetfelder von leicht erreichbarer Stärke nicht orientieren. Es ist auch kein anderes allgemein anwendbares Verfahren bekannt, um bei ihnen homogen orientierte Schichten herstellen zu können. In Sonderfällen kann man durch sorgfältiges Abkühlen einer homogen orientierten nematischen Schicht gut orientierte smektische Schichten erhalten. Die dabei nie ganz zu vermeidenden Inhomogenitäten werden sich aber sicher störend auf die Linienbreite in den Kernresonanzspektren auswirken. Smektische Schichten haben aber andererseits gerade durch die geringe Wirkung des Magnetfeldes auf ihre Orientierung den besonderen Vorteil, daß sich der Winkel zwischen optischer Achse und Magnetfeld beliebig wählen läßt. Ein Verfahren, sie wirklich vollständig homogen auszurichten, wäre deshalb von großem Nutzen.

\section{Lösungen in kristallinflüssigen Phasen und die Ordnungsmatrix}

Mischt man einer kristallinflüssigen Phase Fremdsubstanzen bei, so bleibt der kristallinflüssige $\mathrm{Zu}$ stand innerhalb gewisser Konzentrationsgrenzen erhalten und man erhält damit Lösungen in kristallinflüssigen Phasen. Die maximal erreichbare Konzentration hängt $a b$ von der Art des gelösten Stoffes und von der kristallinflüssigen Substanz. Allgemeine Angaben über ihre Höhe können daher nicht gemacht werden. Es scheint aber so zu sein, daß Substanzen, deren Schmelzpunkt wesentlich höher liegt als der kristallinflüssige Bereich, nur in geringer Menge lösbar sind, weil sie leicht ausfallen.

Die gelösten Moleküle erfahren auf Grund ihrer Anisotropie eine gewisse Orientierung im kristallinflüssigen Lösungsmittel. Sie brauchen keineswegs eine ebenso ausgeprägt langgestreckte Form zu besitzen wie die Moleküle der kristallinflüssigen Substanzen. Im Gegensatz zu den reinen kristallinflüssigen Phasen genügt es aber nun im allgemeinen nicht mehr, sich mit der Orientierung einer einzigen ausgezeichneten molekularen Achse zu befassen, vielmehr sind mehrere Größen notwendig, um die mittlere Orientierung in einer für unsere Zwecke hinreichenden Weise zu kennzeichnen. Diesem Problem wollen wir uns nun zuwenden.

$x_{1}^{\prime}, x_{2}{ }^{\prime}, x_{3}{ }^{\prime}$ sei ein spezielles raumfestes kartesisches Koordinatensystem, dessen $x_{3}{ }^{\prime}$-Achse parallel zur optischen Achse der homogen orientiert angenommenen kristallinflüssigen Schicht liegt. Wir betrachten ein starres Molekül in dieser Schicht, also ein Molekül ohne innere Beweglichkeit. $\xi_{1}, \xi_{2}, \xi_{3}$ bezeichnen die Achsen eines molekülfesten Systems und gleichzeitig die Komponenten eines molekülfesten Vektors. Im raumfesten System bezeichnen wir seine Komponenten mit $x_{1}{ }^{\prime}, x_{2}{ }^{\prime}, x_{3}{ }^{\prime}$.

Die Orientierung der Moleküle in kristallinen Flüssigkeiten ist apolar, die zeitlichen Mittelwerte oder Erwartungswerte $\left\langle x_{i}^{\prime}\right\rangle$ sind deshalb alle gleich Null. $\eta_{1}, \eta_{2}, \eta_{3}$ sei ein zweiter molekülfester Vektor und $y_{1}^{\prime}, y_{2}^{\prime}, y_{3}^{\prime}$ seine Komponenten im raumfesten System. Wir wollen die Mittelwerte $\left\langle x_{i}^{\prime} y_{j}^{\prime}\right\rangle$, also die Mittelwerte der im raumfesten System geltenden Komponenten des molekülfesten Tensors zweiter Stufe berechnen, bzw. sie durch die Komponenten des molekülfesten Systems ausdrücken.

Wegen der Rotationssymmetrie um die optische Achse und wegen der apolaren Orientierung verschwinden alle Mittelwerte $\left\langle x_{i}^{\prime} y_{j}^{\prime}\right\rangle$, für $i \neq j$ und es 
gilt $\left\langle x_{1}^{\prime} y_{1}^{\prime}\right\rangle=\left\langle x_{2}^{\prime} y_{2}^{\prime}\right\rangle$. Aus der Invarianz der Spur folgt

$$
\left\langle x_{1}^{\prime} y_{1}^{\prime}\right\rangle=\left\langle x_{2}{ }^{\prime} y_{2}{ }^{\prime}\right\rangle=\left(\sum_{i} \xi_{i} \eta_{\mathrm{i}}-\left\langle x_{3}{ }^{\prime} y_{3}{ }^{\prime}\right\rangle\right) / 2 .
$$

Bezeichnen wir mit $\Theta_{i}$ die Winkel der $x_{3}{ }^{\prime}$-Achse oder allgemeiner der optischen Achse mit den molekülfesten $\xi_{i}$-Achsen, so gilt

$$
\begin{gathered}
\left\langle x_{3}{ }^{\prime} y_{3}{ }^{\prime}\right\rangle=\sum_{i j} \xi_{i} \eta_{j}\left\langle\cos \Theta_{i} \cos \Theta_{j}\right\rangle, \\
\left\langle x_{1}{ }^{\prime} y_{1}{ }^{\prime}\right\rangle=\left\langle x_{2}{ }^{\prime} y_{2}{ }^{\prime}\right\rangle \\
=\frac{1}{2} \sum_{i} \xi_{i} \eta_{i}-\frac{1}{2} \sum_{i j} \xi_{i} \eta_{j}\left\langle\cos \Theta_{i} \cos \Theta_{j}\right\rangle .
\end{gathered}
$$

Wir können das Ergebnis auch, wie folgt, schreiben

$$
\begin{gathered}
\left\langle x_{3}{ }^{\prime} y_{3}{ }^{\prime}\right\rangle=\frac{1}{3} \sum_{i} \xi_{i} \eta_{i}+\frac{2}{3} \sum_{i j} S_{i j} \xi_{i} \eta_{j}, \\
\left\langle x_{1}{ }^{\prime} y_{1}{ }^{\prime}\right\rangle=\left\langle x_{2}{ }^{\prime} y_{2}{ }^{\prime}\right\rangle=\frac{1}{3} \sum_{i} \xi_{i} \eta_{i}-\frac{1}{3} \sum_{i j} S_{i j} \xi_{i} \eta_{j} .
\end{gathered}
$$

Zur Abkürzung wurde hierbei gesetzt

$$
S_{i j}=\frac{1}{2}\left\langle 3 \cos \Theta_{i} \cos \Theta_{j}-\delta_{i j}\right\rangle .
$$

$\delta_{i j}$ bedeutet das Kronecker-Symbol. Wählen wir ein beliebiges raumfestes kartesisches Koordinatensystem mit den Achsen $x_{i}$ und ist $x_{i}^{\prime}=\sum_{l} r_{i l} x_{l}$, so gilt für die Mittelwerte

$$
\begin{aligned}
\left\langle x_{k} x_{l}\right\rangle & =\sum_{i j} r_{i k} r_{j l}\left\langle x_{i}^{\prime} y_{j}^{\prime}\right\rangle \\
& =\frac{1}{3} \delta_{k l} \sum_{i} \xi_{i} \eta_{i}+\left(r_{3 k} r_{3 l}-\frac{1}{3} \delta_{k l}\right) \sum_{i j} S_{i j} \xi_{i} \eta_{j} .
\end{aligned}
$$

Bezeichnen wir also mit $\alpha_{i}$ die Winkel zwischen den $x_{i}$-Achsen und der optischen Achse, so gilt

$$
\begin{aligned}
\left\langle x_{k} x_{l}\right\rangle= & \frac{1}{3} \delta_{k l} \sum_{i} \xi_{i} \eta_{i} \\
& +\left(\cos \alpha_{k} \cos \alpha_{l}-\frac{1}{3} \delta_{k l}\right) \sum_{i j} S_{i j} \xi_{i} \eta_{j} .
\end{aligned}
$$

Diese Gleichung für die Mittelwerte in einem raumfesten System gilt, sinngemäß übertragen, für jeden molekülfesten Tensor zweiter Stufe; denn jeder Tensor ist durch eine lineare Überlagerung der speziellen Tensoren darstellbar, für die wir die Gleichung hergeleitet haben. Für schiefsymmetrische Tensoren verschwinden die Mittelwerte.

Die Matrix $\left(S_{i j}\right)$ nennen wir Ordnungsmatrix in Analogie zum früher definierten Ordnungsgrad. Sie ist Null für isotrope Flüssigkeiten. Wählt man ein anderes molekülfestes Koordinatensystem mit den Achsen $\xi_{i}^{\prime}$, das mit dem alten durch die orthogonale Transformation $\xi_{i}=\sum_{j} t_{i j} \xi_{j}^{\prime}$ verknüpft ist, so gilt insbesondere auch für $\cos \Theta_{i}=\sum_{j} t_{i j} \cos \Theta_{j}{ }^{\prime}$, wenn wir mit $\Theta_{j}^{\prime}$ den Winkel zwischen der optischen Achse und $\operatorname{der} \xi_{j}^{\prime}$-Achse bezeichnen. Hieraus folgt

$$
S_{i j}=\sum_{l m} t_{i l} t_{j m} S_{l m}^{\prime},
$$

wobei $\left(S^{\prime} \imath_{m}\right)$ die auf das gestrichene System bezogene Ordnungsmatrix ist.

Die Ordnungsmatrix transformiert sich also bei einer Änderung des molekülfesten Koordinatensystems wie ein Tensor zweiter Stufe. Sie ist außerdem reell und symmetrisch und kann deshalb durch eine passende Wahl des molekülfesten Koordinatensystems auf Diagonalform gebracht werden. (Bei innerer Beweglichkeit kann für jeden in sich starren Molekülteil eine eigene Ordnungsmatrix aufgestellt werden. Sie kann nützlich sein, zur Untersuchung der Wechselwirkung innerhalb der starren Teile.)

Die Hauptachsen von $\left(S_{i j}\right)$ definieren ein molekülfestes Koordinatensystem, das bezüglich der zwischenmolekularen Kräfte in der kristallinflüssigen Umgebung ausgezeichnet ist. Ihre Lage wird vermutlich im wesentlichen durch die Dispersionskräfte bestimmt und somit nur wenig von der speziellen kristallinflüssigen Umgebung abhängen. Sie wird annähernd zusammenfallen mit der Hauptachsenlage des Polarisierbarkeitstensors. Da der Polarisierbarkeitstensor oft der Molekülform entspricht, wird dieselbe Lage auch durch den Packungseffekt begünstigt werden. Natürlich haben auch die elektrischen Dipol-DipolKräfte und die Induktionseffekte einen gewissen Einfluß, der besonders dann hervortritt, wenn das betrachtete Molekül ein permanentes elektrisches Dipolmoment hat und seine Polarisierbarkeitsanisotropie gering ist. Es wird sich dann auch eine Abhängigkeit der Hauptachsenlage von der speziellen kristallinflüssigen Trägersubstanz ergeben, weil die Größe und die Richtung des Dipolmoments der Trägersubstanz von Bedeutung ist.

Die Spur $\sum_{i} S_{i i}$ verschwindet. Die Ordnungsmatrix enthält also im allgemeinen 5 voneinander unabhängige Elemente; wenn die Hauptachsen aus Symmetriegründen schon festliegen, sind es nur noch zwei. Die Matrix muß natürlich invariant gegenüber Symmetrieoperationen sein. Ist z. B. eine Symmetrieebene vorhanden, so steht eine der Hauptachsen senkrecht darauf. Ebenso definiert eine zweizählige Symmetrieachse eine Hauptachsenrichtung. Hat das Molekül eine mehr als 2-zählige Symmetrieachse, die hier als $\xi_{3}$ gewählt sei, so ist $\left(S_{i j}\right)$ vollständig auf 
Diagonalform und es gilt $S_{11}=S_{22}=-S_{33} / 2$. In guter Näherung wird das gleiche auch dann gelten, wenn das gelöste Molekül einen so langgestreckten Bau hat wie die Moleküle der kristallinflüssigen Substanzen. Bei der dann zu erwartenden relativ hohen Orientierung der Längsachse kann nämlich die Anisotropie senkrecht dazu näherungsweise vernachlässigt werden. In diesen beiden Fällen wird die Orientierung durch die Angabe eines einzigen Diagonalelements charakterisiert. Zweckmäßigerweise wählt man hierzu das Diagonalelement der ausgezeichneten Richtung, also der Symmetrieachse oder der Längsachse. So verfährt man insbesondere bei den Molekülen der kristallinflüssigen Substanz selbst. Man bezeichnet dieses Element dann einfach als Ordnungsgrad, wie wir es im vorigen Abschnitt getan haben.

Über die praktisch vorkommenden Größen der Hauptachsen Elemente $S_{i i}$ lassen sich noch keine allgemeinen Angaben machen, da bisher hierüber nur wenige experimentelle Daten vorliegen. $S_{i i}$ ist sicher stark von den Eigenschaften des gelösten Moleküls, sowie der Temperatur und dem Ordnungsgrad der umgebenden kristallinflüssigen Substanz, aber vermutlich von deren spezifischen molekularen Eigenschaften nur wenig abhängig. Für die Vorzeichen der Diagonalelemente gilt vermutlich die allgemeine Regel, daß bei nematischen und smektischen Flüssigkeiten immer dasjenige Element positiv ist, welches zur Achse mit der relativ größten Polarisierbarkeit gehört und dementsprechend negativ dasjenige mit der kleinsten Polarisierbarkeit. Bei cholesterinischen Flüssigkeiten verhält es sich gerade umgekehrt.

\section{Der Spin-Hamilton-Operator für die inner- molekulare Wechselwirkung in kristallinen Flüssigkeiten}

Wir betrachten wieder ein starres Molekül, das sich in einer kristallinflüssigen Schicht befindet. Wir nehmen an, daß die Zeeman-Terme groß sind gegenüber allen übrigen Wechselwirkungstermen, so daß die Mischung von Spin-Funktionen, die zu verschiedenen magnetischen Quantenzahlen gehören, nicht berücksichtigt werden braucht.

Der vollständige Spin-Намıтол-Operator für ein festorientiertes starres Molekül läßt sich folgendermaßen schreiben:

$$
\begin{aligned}
\boldsymbol{H}= & -\hbar \sum_{p} \sum_{k} \gamma_{p} H_{k} \boldsymbol{I}_{k}^{p}+\hbar \sum_{p} \sum_{k l} \gamma_{p} \varrho_{p}^{k l} H_{k} \boldsymbol{I}_{l}^{p} \\
& +\hbar^{2} \sum_{p q} \sum_{l k} B_{l k}^{p q} \boldsymbol{I}_{l}^{p} \boldsymbol{I}_{k}^{p} .
\end{aligned}
$$

Hierbei laufen die Indizes $p$ und $q$ über die wechselwirkenden Kerne, $l$ und $k$ laufen von 1 bis $3 . H_{k}$ ist die $x_{k}$-Komponente des angelegten Magnetfeldes $H_{0}$. Es wird ein raumfestes kartesisches Koordinatensystem mit den Achsen $x_{1}, x_{2}, x_{3}$ zugrunde gelegt. $\boldsymbol{I}_{\mathrm{p}}$ ist der Spin-Operator des $p$-ten Kerns. $\boldsymbol{I}_{k}^{p}$ die $x_{k}$ Komponente davon, $\hbar \gamma_{p} \mathbf{I}_{p}$ sein magnetisches Dipolmoment. $\varrho_{k l}^{p}$ sind die Elemente des Abschirmungstensors, der die chemische Verschiebung beschreibt. Die Konstanten $B_{l k}^{p q}$ erfassen für $p \neq q$ die direkte und die indirekte magnetische Dipol-Dipol-Wechselwirkung und für $p=q$ die Quadrupolwechselwirkung.

Die $x_{3}$-Richtung unseres Koordinatensystems legen wir parallel zum Magnetfeld. Unter der Voraussetzung des starken Magnetfeldes können wir einige Summanden in (6) vernachlässigen; nämlich alle diejenigen, welche die $x_{3}$-Komponente des Gesamtspins ändern. Wir finden sie leicht, indem wir die Operatoren $\boldsymbol{I}_{1}^{p}$ und $\boldsymbol{I}_{2}^{p}$ durch $\mathbf{I}_{+}^{p}=\mathbf{I}_{1}^{p}+i \mathbf{I}_{2}^{p}$ und $\mathbf{I}_{-}^{p}=$ $\mathbf{I}_{1}^{p}-i \mathbf{I}_{2}^{p}$ ausdrücken. Werden diese Operatoren auf eine Eigenfunktion des Operators $\sum_{p} \boldsymbol{I}_{3}^{p}$ angewendet, so ändern sie bekanntlich den Eigenwert um + bzw. -1 oder machen die Funktion zu Null. Es ist:

$$
\begin{aligned}
& \boldsymbol{I}_{1}^{p} \boldsymbol{I}_{1}^{q}=\frac{1}{4}\left(\boldsymbol{I}_{+}^{p} \boldsymbol{I}_{+}^{q}+\boldsymbol{I}_{-}^{p} \boldsymbol{I}_{-}^{q}+\boldsymbol{I}_{+}^{p} \boldsymbol{I}_{-}^{q}+\boldsymbol{I}_{-}^{p} \boldsymbol{I}_{+}^{q}\right), \\
& \boldsymbol{I}_{2}^{p} \boldsymbol{I}_{2}^{q}=-\frac{1}{4}\left(\boldsymbol{I}_{+}^{p} \boldsymbol{I}_{+}^{q}+\boldsymbol{I}_{-}^{p} \boldsymbol{I}_{-}^{q}-\boldsymbol{I}_{+}^{p} \boldsymbol{I}_{-}^{q}-\boldsymbol{I}_{-}^{p} \boldsymbol{I}_{+}^{q}\right), \\
& \boldsymbol{I}_{1}^{p} \boldsymbol{I}_{2}^{q}=\frac{1}{4} i\left(\boldsymbol{I}_{+}^{p} \boldsymbol{I}_{+}^{q}-\boldsymbol{I}_{-}^{p} \boldsymbol{I}_{-}^{q}-\boldsymbol{I}_{+}^{p} \boldsymbol{I}_{-}^{q}+\boldsymbol{I}_{-}^{p} \boldsymbol{I}_{+}^{q}\right), \\
& \boldsymbol{I}_{2}^{p} \boldsymbol{I}_{1}^{q}=\frac{1}{4} i\left(\boldsymbol{I}_{+}^{p} \boldsymbol{I}_{+}^{q}-\boldsymbol{I}_{-}^{p} \boldsymbol{I}_{-}^{q}+\boldsymbol{I}_{+}^{p} \boldsymbol{I}_{-}^{q}-\boldsymbol{I}_{-}^{p} \boldsymbol{I}_{+}^{q}\right) .
\end{aligned}
$$

Hiermit und mit $B_{k l}^{p q}=B_{l k}^{p q}$ ergibt sich für den Hamilton-Operator in der betrachteten Näherung

$$
\begin{aligned}
& \boldsymbol{H}=-\hbar \sum_{p} \gamma_{p}\left(1-\varrho_{33}^{p}\right) H_{0} \boldsymbol{I}_{3}^{p} \\
& +\hbar^{2} \sum_{p q}\left\{\frac{1}{4}\left(B_{11}^{p q}+B_{22}^{p q}\right)\left(\boldsymbol{I}_{+}^{p} \boldsymbol{I}_{-}^{q}+\boldsymbol{I}_{-}^{p} \boldsymbol{I}_{+}^{q}\right)+B_{33}^{p q} \boldsymbol{I}_{3}^{p} \boldsymbol{I}_{3}^{q}\right\} \\
& =-\hbar \sum_{p} \gamma_{p}\left(1-\varrho_{33}^{p}\right) H_{0} \mathbf{I}_{3}^{p} \\
& +\hbar^{2} \sum_{p q}\left\{\frac{1}{2}\left(B_{11}^{p q}+B_{22}^{p q}\right) \mathbf{I}_{p} \mathbf{I}_{q}\right. \\
& \left.+\left[B_{33}^{p q}-\frac{1}{2}\left(B_{11}^{p q}+B_{22}^{p q}\right)\right] \mathbf{I}_{3}^{p} \mathbf{I}_{3}^{q}\right\} .
\end{aligned}
$$

Diese Gleichungen gelten also bei einer fest vorgegebenen Orientierung des Moleküls. Wir wollen nun, wie man es bei gewöhnlichen Flüssigkeiten tut, über die verschiedenen Orientierungen mitteln, müssen aber dabei die zur kristallinflüssigen Ordnung gehörenden statistischen Gewichte beachten. Wir bezeichnen hierzu die auf das molekülfeste System bezogenen Abschirmungs- und Kopplungskonstanten mit $\sigma_{l k}^{p}$ und $A_{l k}^{p q}$. Sie sind bei fester Orientierung 
identisch mit den konstanten $\varrho_{l k}^{p}$ bzw. $B_{l k}^{p q}$, wenn raum- und molekülfestes Koordinatensystem zusammenfallen. Außerdem benutzen wir die folgenden Abkürzungen

$$
\begin{array}{rlrl}
\sigma_{p} & =\frac{1}{3} \sum_{l} \sigma_{l l}^{p} ; & J_{p q}=\hbar \frac{2}{3} \sum_{l} A_{l l}^{p q} ; \\
\delta_{p}=\sum_{l k} S_{l k} \sigma_{l k}^{p} ; & B_{p q}=\hbar \sum_{l k} A_{l k}^{p q} S_{l k} .
\end{array}
$$

Nach Gl. (5) erhalten wir damit für die Mittelwerte im raumfesten System, wenn $\alpha$ der Winkel zwischen Magnetfeld oder $x_{3}$-Richtung und der optischen Achse ist

$$
\begin{aligned}
\left\langle\varrho_{33}^{p}\right\rangle & =\sigma_{p}+\frac{1}{3}\left(3 \cos ^{2} \alpha-1\right) \delta_{p}, \\
\hbar\left\langle B_{33}^{p q}\right\rangle & =\frac{1}{2} J_{p q}+\frac{1}{3}\left(3 \cos ^{2} \alpha-1\right) B_{p q}, \\
\frac{1}{2} \hbar\left\langle B_{11}^{p q}+B_{22}^{p q}\right\rangle & =\frac{1}{2} J_{p q}-\frac{1}{6}\left(3 \cos ^{2} \alpha-1\right) B_{p q} .
\end{aligned}
$$

Für den Hamilton-Operator ergibt sich damit aus (7)

$$
\begin{aligned}
& \boldsymbol{H}=-\hbar \sum_{p} \gamma_{p}\left(\mathbf{l}-\sigma_{p}\right) H_{0} \mathbf{I}_{3}^{p}+\frac{1}{2} \hbar \sum_{p q} J_{p q} \mathbf{I}_{p} \mathbf{I}_{q} \\
& +\frac{1}{3} \hbar\left(3 \cos ^{2} \alpha-1\right)\left\{\sum_{p} \gamma_{p} \delta_{p} H_{0} \mathbf{I}_{3}^{p}\right. \\
& \left.+\frac{1}{2} \sum_{p q} B_{p q}\left(3 \boldsymbol{I}_{3}^{p} \mathbf{I}_{3}^{q}-\mathbf{I}_{p} \mathbf{I}_{q}\right)\right\} .
\end{aligned}
$$

Im Sonderfall $S_{11}=S_{22}=-S_{33} / 2$ und $S_{l k}=0$ für $l \neq k$ gilt

$$
\begin{aligned}
\delta_{p} & =S_{33}\left(\sigma_{33}^{p}-\frac{1}{2}\left(\sigma_{11}^{p}+\sigma_{22}^{p}\right)\right), \\
B_{p q} & =\hbar S_{33}\left(A_{33}^{p q}-\frac{1}{2}\left(A_{11}^{p q}+A_{22}^{p q}\right)\right) .
\end{aligned}
$$

Man kann dann in Gl. (10) den Ordnungsparameter $S_{33}$ vor die geschweifte Klammer ziehen.

Gl. (10) gilt zunächst nur für ein starres Molekül. Liegen verschiedene Rotationsisomere vor, so muß man die Gleichung für jedes Rotationsisomer gesondert aufstellen. Bei hinreichend rascher Umwandlung zwischen den Rotationsisomeren sind die einzelnen Gleichungen mit den entsprechenden statistischen Gewichten zu addieren. Man erhält so für $\delta_{p}$ den Mittelwert

$$
\sum_{l k}\left\langle S_{l k} \varrho_{l k}^{p}\right\rangle=\sum_{l k}\left\langle\left(3 \cos \Theta_{l} \cos \Theta_{k}-\delta_{l k}\right) \varrho_{l k}^{p}\right\rangle / 2
$$

und für $B_{p q}$ den Mittelwert

$$
\sum_{l k} \hbar\left\langle S_{l k} A_{l k}^{p q}\right\rangle=\hbar \sum_{l k}\left\langle\left( 3 \cos \Theta_{l} \cos \Theta_{k}\right.\right.
$$

$$
\left.\left.-\delta_{l k}\right) A_{l k}^{p q}\right\rangle / 2 .
$$

Sind die verschiedenen Rotationsisomeren gleichwertig bezüglich der Orientierung, d. h. kann man in allen Rotationsisomeren ein Koordinatensystem wählen, das zu denselben $S_{l k}$ führt, so gilt

$$
\left\langle S_{l k} A_{l k}^{p q}\right\rangle=S_{l k}\left\langle A_{l k}^{p q}\right\rangle \text { und }\left\langle S_{l k} \varrho_{l k}^{p}\right\rangle=S_{l k}\left\langle\varrho_{l k}^{p}\right\rangle \text {. }
$$

Man kann dann also die innere Bewegung getrennt von der äußeren betrachten.

Die ersten beiden Summen in Gl. (10) enthalten den isotropen Anteil der Wechselwirkung, also den isotropen Teil der Abschirmung und der indirekten Spin-Spin-Wechselwirkung. Dieser Anteil bleibt allein übrig, wenn wir alle $S_{l k}$ gegen Null gehen lassen, also zur isotropen Flüssigkeit übergehen, oder wenn das Magnetfeld einen Winkel von $55^{\circ}$ mit der optischen Achse bildet, weil dann $3 \cos ^{2} \alpha=1$ ist. Den isotropen Teil wird man in manchen Fällen gegenüber dem anisotropen Teil vernachlässigen dürfen, er ist aber sicher immer dann von Bedeutung, wenn die Orientierung schwach ist.

Die erste Summe in der geschweiften Klammer erfaßt den anisotropen Anteil der Abschirmung. Die einzelnen Summanden sind proportional zu $H_{0} \mathbf{I}_{3}^{p}$. Sie haben also den gleichen Einfluß auf das Spektrum wie eine Änderung der $\sigma_{p}$ im isotropen Teil um $\frac{1}{3}\left(3 \cos ^{2} \alpha-1\right) \delta_{p}$. Die Wirkung einer unterschiedlichen Abschirmung gleicher Kerne oder, anders ausgedrückt, einer unterschiedlichen chemischen Verschiebung ist direkt am Aussehen der Spektren zu erkennen. Kann man nämlich die Verschiebung innerhalb der Gruppen gleicher Kerne vernachlässigen, so sind die Spektren der einzelnen Kerngruppen in sich symmetrisch. Treten aber merkliche Unterschiede in der Verschiebung auch nur bei einer Kernsorte auf, so können die Spektren aller Kerngruppen ihre Symmetrie verlieren.

Die letzte Summe in Gl. (10) beschreibt die anisotrope Spin-Spin-Wechselwirkung und die Quadrupolwechselwirkung. Auf diese beiden stärksten Wechselwirkungen wollen wir nun näher eingehen und dann die Frage der absoluten Vorzeichenbestimmung der Kopplungskonstanten diskutieren.

\section{a) Die anisotrope Spin-Spin-Wechselwirkung}

Die anisotrope Spin-Spin-Wechselwirkung enthält die gesamte direkte Dipol-Dipol-Wechselwirkung und den anisotropen Teil der indirekten Spin-SpinWechselwirkung. Letztere wird in unseren kristallinen Flüssigkeiten meist keine Bedeutung haben, da sie durch die direkte Wechselwirkung verdeckt wird. Wir gehen deshalb in unserer Diskussion nicht näher darauf ein und betrachten nur die direkte DipolDipol-Wechselwirkung. 
In Gl. (10) gehören zur betrachteten Wechselwirkung die Koeffizienten $B_{p q}$ mit $p \neq q$. Für diese gilt ${ }^{4,5}$ unter Voraussetzung starrer Moleküle

$$
\begin{aligned}
B_{p q} & =\hbar \sum_{l k} S_{l k} A_{l k}^{\gamma q}=\hbar \frac{\gamma_{p} \gamma_{q}}{2 r_{p q}^{3}} \sum_{l k} S_{l k}\left(\delta_{l k}-\frac{3}{r_{p q}^{2}} \xi_{l}^{p q} \xi_{k}^{p q}\right) \\
& =-\hbar \frac{3 \gamma_{q} \gamma_{p}}{2 r_{p q}^{5}} \sum_{l k} S_{l k} \xi_{l}^{p q} \xi_{k}^{p q} ; \quad p \neq q .
\end{aligned}
$$

Wir haben die Achsen des molekülfesten Koordinatensystems wieder mit $\xi_{l}$ bezeichnet. $\xi_{l}^{p q}$ sind die Komponenten des Ortsvektors $\mathfrak{r}_{p q}$ von $p$ nach $q$. Im Sonderfall $S_{11}=\mathrm{S}_{22}=-S_{33} / 2$ und alle $S_{l k}=0$ für $l \neq k$ gilt

$$
B_{p q}=-\hbar \frac{3 \gamma_{p} \gamma_{q}}{4 r_{p q}^{3}} S_{33}\left(3 \cos ^{2} \beta_{p q}-1\right) .
$$

Hierbei haben wir mit $\beta_{p q}$ den Winkel zwischen Kernverbindungsachse und $\xi_{3}$-Richtung bezeichnet. Diese Beziehung haben LippmanN ${ }^{6}$ und $\mathrm{Weber}^{7}$ zur Deutung von Kernresonanzuntersuchungen an nematischen und smektischen Flüssigkeiten verwendet.

Es steht uns frei, für jedes Kernpaar das molekülfeste Koordinatensystem so zu wählen, daß die $\xi_{3}$ Richtung mit der Kernverbindungsachse zusammenfällt. Bezeichnen wir die Elemente der Ordnungsmatrix, die zu diesem speziellen System gehören mit $S_{l k}^{p q}$, dann gilt

$$
B_{p q}=-\hbar \frac{3 \gamma_{p} \gamma_{q}}{2 r_{p q}^{3}} S_{33}^{p q} .
$$

Diese Beziehung gilt auch noch, wenn eine innere Bewegung zwar möglich ist, der Kernabstand $r_{p q}$ dabei aber nicht geändert wird. Man erhält also in jedem Fall aus $B_{p q}$ bei bekanntem Kernabstand sofort die mittlere Orientierung oder den Ordnungsgrad der betrachteten Kernachse (es ist daher möglich, für jeden in sich starren Molekülteil eine eigene Ordnungsmatrix zu bestimmen). Umgekehrt kann man bei bekannter Orientierung auch Kernabstände bestimmen. Im allgemeinen werden zur Lagebestimmung eines Kernes die Kopplungen mit 3 Kernen schon bekannter Lage ausreichen. Diese 3 Kerne können auch ausreichen, um bei einem starren Molekül mit ebener Symmetrie die Orientierung festzulegen.

Wir wollen noch einen Sonderfall der inneren Bewegung betrachten. Wir nehmen an, daß der Kern $q$

4 A. Abragam, The Principles of Nuclear Magnetism, Oxford University Press, Oxford 1961.

5 N. Blogmbergen, E. M. Purcel u. R. V. Pound, Phys. Rev. 73, 679 [1948]. sich um eine im Molekülrumpf feste Achse in einem 3- oder mehrfach periodischen Potential bewegt. Der Kern $p$ liege auf dieser Achse. Die auf den Molekülrumpf bezogene Ordnungsmatrix soll durch die Drehung nicht beeinflußt werden. Lassen wir hier die $\xi_{3^{-}}$. Richtung mit der Drehachse zusammenfallen, so gilt für $B_{p q}$ wieder die Gl. (12). Sie gilt auch für den Fall, daß $p$ und $q$ sich zusammen um die $\xi_{3}$-Richtung in einem 3- oder mehrfach periodischen Potential bewegen.

\section{b) Die Quadrupolwechselwirkung}

In der letzten Summe von Gl. (10) beschreiben die Glieder mit $p=q$ die Quadrupolwechselwirkung. Sie ist also gegeben durch

$$
\mathbf{H}_{\mathrm{Q}}=\frac{1}{6} \hbar\left(3 \cos ^{2} \alpha-1\right) \sum_{p} B_{p p}\left(3 \mathbf{I}_{3}^{p} \mathbf{I}_{3}^{p}-I^{p}\left(I^{p}+1\right)\right) .
$$

Hierin bedeutet $I^{p}$ die Spin-Quantenzahl im Gegensatz zum Spin-Operator $\boldsymbol{I}_{p}$. Für die Konstanten $B_{p p}$ gilt 4,8

$$
\hbar B_{p p}=\frac{e Q_{p}}{2 I^{p}\left(2 I^{p}-1\right)} \sum_{l k} S_{l k} V_{l k}^{p} .
$$

Hierbei ist $e$ die Elementarladung und $e Q_{p}$ das Quadrupolmoment des $p$-ten Kerns. $V^{p}$ ist das elektrostatische Potential, das auf den Kern $p$ einwirkt und $V_{k l}^{p}$ steht für die Ableitung $\partial^{2} V^{p} / \partial \xi_{k} \partial \xi_{l}$ am Kernort.

Für $S_{11}=S_{22}=-S_{33} / 2$ und $S_{l k}=0$ für $l \neq k$ wird

$$
\hbar B_{p p}=\frac{3 e Q_{p}}{4 I p\left(2 I^{p}-1\right)} V_{33}^{p} S_{33} .
$$

Dieselbe Gleichung gilt auch für den Fall, daß der Tensor $V_{l k}^{p}$ rotationssymmetrisch ist und die $\xi_{3}$-Achse parallel zu dieser Symmetrieachse gelegt wird.

Die Quadrupolaufspaltung ist in kristallinen Flüssigkeiten bisher noch nicht beobachtet worden. Es sei deshalb auf ein Beispiel hingewiesen, bei dem diese Aufspaltung vermutlich leicht $\mathrm{zu}$ beobachten ist. Einige der p-Alkyl- und p-Alkoxybenzoesäurederivate bilden nematische kristallinflüssige Phasen. Die Substanzen können leicht an der OH-Gruppe deuteriert werden. Das Deuteron wechselt in der flüssigen Substanz seinen Partner so häufig, daß die direkte DipolDipol-Wechselwirkung auch in der nematischen Phase vollkommen herausgemittelt wird. Es bleibt

\footnotetext{
6 H. Lippmann, Ann. Phys., Lpz. 2, 287 [1958].

7 K. H. Weber, Ann. Phys., Lpz. 3, 1 [1959].

8 R. V. Pound, Phys. Rev. 78, 685 [1950].
} 
allein die Quadrupolaufspaltung, die hier aus Gln. (14) und (16) berechnet werden kann. Man beobachtet demnach zwei Linien im Abstand (3/2) $e Q V_{33} S_{33}$. Als $\xi_{3}$-Richtung wählen wir die Richtung der Paraachse. Sie wird annähernd zusammenfallen mit der O-D-Bindungsrichtung. Übernimmt man zur Abschätzung die Quadrupolkopplungskonstanten von $\mathrm{D}_{2} \mathrm{O}$, die in einem Einkristall von $\mathrm{Li}_{2} \mathrm{SO}_{4}$ gemessen ${ }^{9}$ wurden, so folgt $\Delta v=355 \mathrm{kHz} \cdot S_{33}$. $S_{33}$ liegt sicher nahe bei 0,6 . Es ist demnach eine Aufspaltung von etwa $200 \mathrm{kHz}$ zu erwarten.

\section{c) Die Vorzeichenbestimmung der Spin-Spin- Kopplungskonstanten}

Im Gegensatz zum Vorzeichen der chemischen Verschiebung, kann das Vorzeichen der Spin-Spin-Kopplungskonstanten nicht direkt aus dem Spektrum entnommen werden. Auch in kristallinen Flüssigkeiten gilt trotz der Orientierung das gleiche wie in isotropen Flüssigkeiten. Kehrt man das Vorzeichen aller Spin-Spin-Kopplungskonstanten um, so ändern sich die Übergangsenergien und Übergangswahrschein. lichkeiten nicht. Gelingt es aber, das Vorzeichen einer einzigen Kopplungskonstanten nach anderen Überlegungen zu ermitteln, so ergeben sich die Vorzeichen aller übrigen wirksamen Kopplungsparameter im allgemeinen eindeutig aus dem Spektrum. Bei nur ungefähr bekannter Molekülstruktur lassen sich leicht die Vorzeichen wenigstens einiger der direkten DipolDipol-Kopplungen angeben, sofern genügend über die bevorzugte Orientierung des Moleküls bekannt ist. Das Problem der Vorzeichenbestimmung reduziert sich damit auf die Frage, welche Molekülorientierungen in kristallinen Flüssigkeiten bevorzugt werden oder anders formuliert, welche Diagonalelemente der Ordnungsmatrix positiv sind.

Die Vorzeichen der Elemente $S_{l k}$ sind zunächst ebenso unbekannt wie die Vorzeichen der Kopplungsparameter $B_{p q}$, aus denen sie berechnet werden. In Einzelfällen können sie sich aber daraus ergeben, daß der Betrag negativer Diagonalelemente nicht größer als 0,5 werden kann, während er bei positiven bis zu 1 ansteigen kann. Ist also eines der Diagonalelemente $S_{l l}$ dem Betrag nach größer als 0,5 , so muß es stets positiv sein. Bei gelösten Molekülen sind die Diagonalelemente sicher selten so groß, daß diese Überlegung von Nutzen sein kann. In manchen Fällen kann die Untersuchung der

9 S. Kedutat u. R. V. Pound, J. Chem. Phys. 26, 708 [1957]. chemischen Verschiebung weiterhelfen, deren Vorzeichen sich eindeutig aus dem Spektrum ergibt. Gelingt es z.B. den anisotropen Teil der chemischen Verschiebung eines an einen Benzolring gebundenen Protons zu bestimmen, so können schon einfache theoretische Überlegungen zeigen, welche bevorzugte Orientierung die Ringebene hat. Auch die weiter oben angegebene Regel wird oft von Nutzen sein, denn die Polarisierbarkeiten eines Moleküls lassen sich leicht aus seiner Struktur näherungsweise herleiten. Schließlich wird man gelegentlich auch auf Grund anderer Messungen etwa durch UV-Messungen mit polarisiertem Licht die Orientierung experimentell festlegen können.

Die Betrachtungen zeigen, daß die absolute Bestimmung der Molekülorientierung in den meisten Fällen möglich sein wird. Die absolute Bestimmung der Vorzeichen der Kopplungskonstanten, insbesondere der indirekten Spin-Spin- und Quadrupolkopplung, ist dann damit ebenfalls grundsätzlich durchführbar.

\section{Bemerkungen zur Linienbreite in kristallinen Flüssigkeiten und Intensitätsbetrachtungen}

Die Spin-Gitterrelaxationszeiten in kristallinen Flüssigkeiten liegen, der Struktur entsprechend, sicher zwischen denen von Festkörpern und isotropen Flüssigkeiten. Sie sind also insbesondere bei quadrupolfreien Kernen verhältnismäßig lang und lassen geringe Linienbreiten zu. Eine störende Linienverbreiterung, selbst unter besten experimentellen Bedingungen, kann dann nur durch lange Korrelationszeiten für die Translations- und Rotationsbewegung der Moleküle verursacht werden.

Die Translationsbewegung der Moleküle in nematischen und cholesterinischen Flüssigkeiten wird durch die kristallinflüssige Ordnung, die ja hier nur in der Ausrichtung der Moleküle besteht, nicht durch zusätzliche Potentiale beeinträchtigt. Die Beweglichkeit der Moleküle entspricht also am Umwandlungspunkt etwa der Beweglichkeit in der darüberliegenden isotropen Phase und wird die normale Temperaturabhängigkeit zeigen. In smektischen Flüssigkeiten ist die Translationsbewegung in Richtung der optischen Achse zusätzlich behindert. Es ist hierbei ein für diese Flüssigkeiten charakteristisches Potential zu überwinden, das die Anordnung der Moleküle in äquidistanten Ebenen erzwingt. Die Bewegung innerhalb dieser Ebenen bleibt aber unbehindert. Sie 
reicht aus, um die zwischenmolekulare Dipol-DipolWechselwirkung auf den fast verschwindenden Mittelwert zu reduzieren und die Korrelationszeit kurz zu halten.

Die Rotationsbewegung der Moleküle ist in allen kristallinen Flüssigkeiten durch das Potential behindert, welches zu der für sie charakteristischen Orientierung der Moleküle gehört. Dieses Potential ist vermutlich für die bei nematischen und smektischen Flüssigkeiten beobachtete außergewöhnliche Erhöhung der dielektrischen Relaxationszeiten ${ }^{10}$ um 2 bis 3 Größenordnungen allein verantwortlich. Die Häufigkeit der Orientierungssprünge um kleine Winkel oder die Debresche Konstante der „inneren Reibung " wird in kristallinen Flüssigkeiten trotzdem noch etwa gleich der von isotropen Flüssigkeiten sein.

Die dielektrische Relaxationszeit gehört zur polaren Einstellung, während für unsere Betrachtungen die apolare Einstellung wichtig ist. Die zur apolaren Einstellung gehörende Korrelationszeit wird sicher weit weniger durch das zusätzliche Potential beein. flußt. Sie wird also noch nahe bei der Korrelations. zeit von den zugehörigen isotropen Flüssigkeiten lie gen. Im übrigen sei erwähnt, daß die beobachteter dielektrischen Relaxationszeiten fast alle kleiner als $10^{-6} \mathrm{sec}$ sind. Speziell für die nematische Phase von Azoxyanisol ${ }^{10}$ ist sie sogar nahe bei $10^{-8}$ sec.

Die für Kernresonanzen geltende Korrelationszeit ist also in günstigen Fällen, wie z. B. beim Azoxy. anisol, sicher kurz genug, um Linienbreiten von wenigen Hertz zu ermöglichen. Außerdem ist hervorzuheben, daß die in kristallinen Flüssigkeiten gelösten Moleküle oft nur verhältnismäßig schwach orientiert werden und dementsprechend ihre Rotationsbewegung kaum beeinträchtigt wird.

Ganz allgemein darf man nach unseren Überlegungen also annehmen, daß die Spektren der nematischen und smektischen Flüssigkeiten sich aus noch verhältnismäßig scharfen Linien zusammensetzen, wenn die zugehörigen isotropen Flüssigkeiten unmittelbar über dem Klärpunkt scharfe Linien aufweisen. Dieselbe Aussage gilt mit noch größerer Wahrscheinlichkeit für die Spektren der gelösten Moleküle. Es sind natürlich homogen ausgerichtete Schichten erforderlich und außerdem eine gut kon-

10 W. Maier u. G. Meier, Z. Naturforschg. 16 a, 1200 [1961].

11 H. Lippmann u. K. H. Weber, Ann. Phys., Lpz. 20, 265 [1957]. stante und über die ganze Schicht einheitliche Temperatur, weil die mittlere Orientierung der Moleküle temperaturabhängig ist.

In cholesterinischen Flüssigkeiten sind die Verhältnisse komplizierter. Sie orientieren sich im Magnetfeld, wie erwähnt, vermutlich immer so, daß die optische Achse senkrecht zum Feld steht, die Ebenen gleicher Orientierung also parallel zur Feldrichtung. Für die innermolekulare Wechselwirkung ist deshalb neben der betrachteten Winkelschwankung die Diffusion in Richtung der optischen Achse maßgebend oder genauer, die Zeit, die im Mittel gebraucht wird, um die Entfernung zwischen zwei benachbarten Ebenen mit der gleichen Molekülorientierung zu durchlaufen. Der Abstand dieser Ebenen beträgt im allgemeinen einige $0,1 \mu$. Die Zeit, die von den Molekülen der cholesterinischen Substanz zum Durchwandern dieser Strecke gebraucht wird, ist vermutlich groß gegen die in Hertz gemessenen reziproken Linienaufspaltungen, und man kann die Diffusion hier näherungsweise vernachlässigen. Die Ebenen mit gleicher Molekülorientierung liefern dann gleiche Spektren, die dem Spektrum einer nematischen Schicht entsprechen, deren optische Achse parallel zur Vorzugsrichtung in diesen Ebenen liegt. Das Gesamtspektrum einer cholesterinischen Flüssigkeit kann demnach aufgefaßt werden als eine Überlagerung von solchen nematischen Spektren. Es wird sich ein breites Spektrum ohne besondere Feinstruktur ergeben, das allenfalls in der Mitte eine relativ scharfe Spitze hat, die durch die Spektren der Ebenen gebildet wird, deren Vorzugsrichtung einen Winkel von annähernd $55^{\circ}$ mit dem Magnetfeld bildet. Nach Gl. (10) verschwindet nämlich für $\alpha=55^{\circ}$ die gesamte anisotrope Wechselwirkung, so daß die Ebenen schmale scharfe Signale liefern. Bei gelösten Molekülen kann die Aufspaltung sehr klein und die Diffusionsgeschwindigkeit größer sein. In Sonderfällen kann man daher vielleicht auch in cholesterinischen Phasen scharfe Lösungsspektren erhalten.

Protonenresonanzspektren von kristallinen Flüssigkeiten sind schon mehrfach experimentell untersucht worden (siehe z. B. ${ }^{6,7,11-13}$ ), aber nur mit geringem Auflösungsvermögen. Die meisten Messun-

12 W. R. Runyan u. A. W. Nolle, J. Chem. Phys. 27, 1081 [1957].

13 P. Jain, J. C. Lee u. R. D. Spence, J. Chem. Phys. 20, 265 [1955]. 


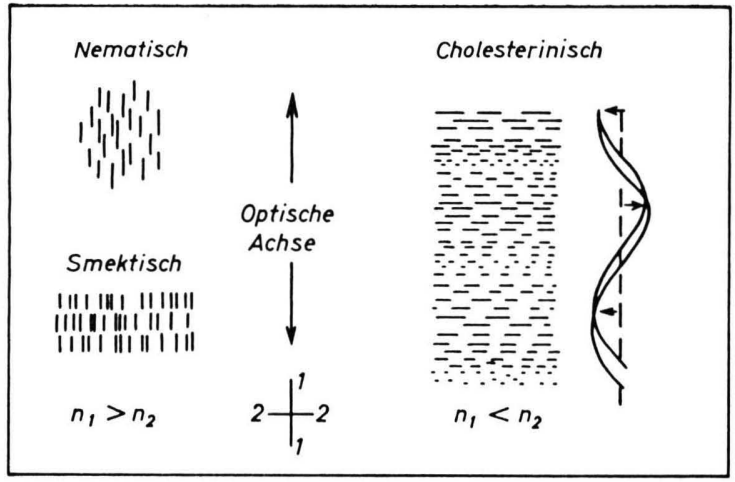

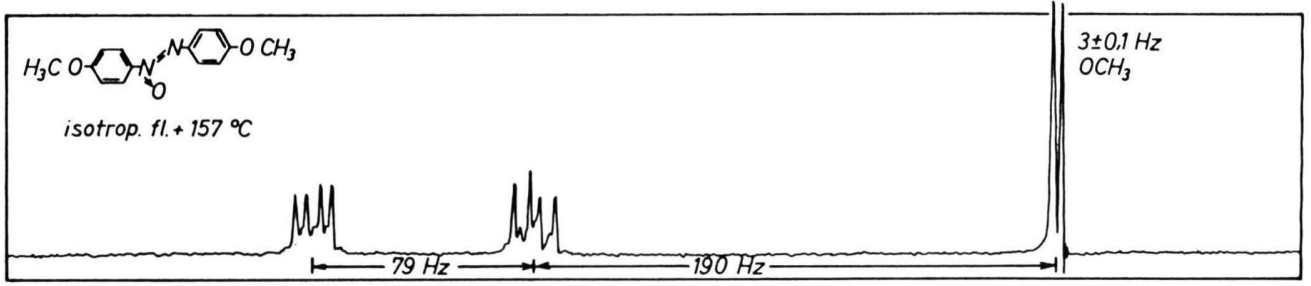

Abb. 2. Azoxyanisol, PR-Spektrum der isotropflüssigen Phase.

Abb. 1. Schematische Strukturbilder der kristallinflüssigen Phasen. Die Moleküle sind durch Striche dargestellt. Bei der cholesterinischen Phase ist ihre Projektion in die Zeichenebene wiedergegeben. Das gewundene Band veranschaulicht die Verdrillung.

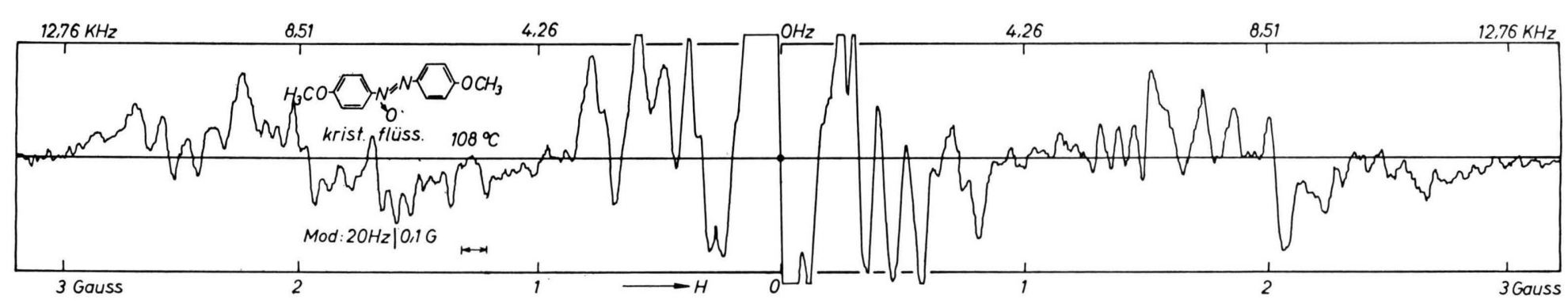

Abb. 3. Azoxyanisol, PR-Spektrum der nematischen Phase (Ableitungskurve).

Abb. 4. Azoxyanisol, PR-Spektrum des Kristalls (Ableitungskurve).

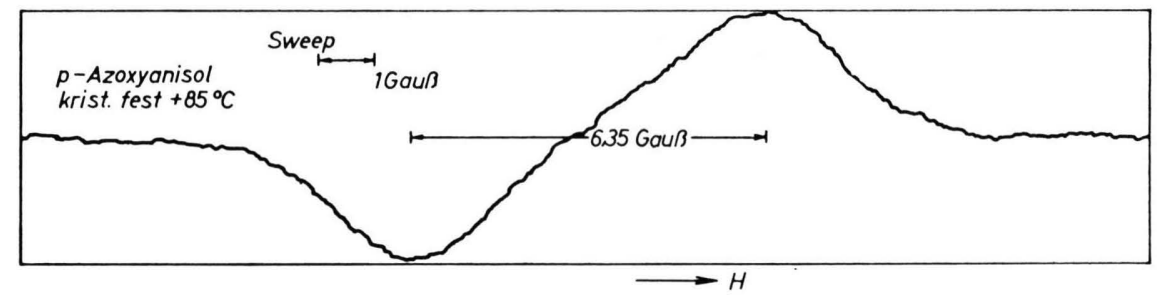


gen wurden an der nematischen Phase von Azoxyanisol durchgeführt; besonders eingehend wurde dieses Spektrum von LippmanN ${ }^{6,11}$ und $\mathrm{W}_{\text {EBER }}{ }^{7}$ untersucht. Die bisher veröffentlichten Spektren zeigen nur wenig Feinstruktur. Im etwa 5 Gauß breiten $\mathrm{Ab}$ sorptionsgebiet des Azoxyanisols z. B. wurde eine Triplettstruktur ohne weitere Feinheiten gefunden.

Angeregt durch die obigen Überlegungen haben wir die Untersuchungen am Azoxyanisol noch einmal aufgegriffen und versucht, hochaufgelöste Spektren zu erhalten. Es ergaben sich in der Tat in der nematischen Phase Spektren mit einer sehr komplizierten Feinstruktur. Abb. 2, 3 und 4 zeigen die mit einem $60 \mathrm{MHz}-\mathrm{V}$ a r i a n - Kernresonanzspektrometer (DP 60) von ENGLeRT aufgenommenen Spektren der drei kondensierten Phasen. Vermutlich wird man bei noch größerem Auflösungsvermögen ein noch komplizierteres Spektrum der nematischen Phase erhalten, aber eine vollständige Auflösung wird nicht möglich sein. Den 14 Protonen des Azoxyanisols entsprechen nämlich $2^{14}$ ZEEMAN-Terme und die Zahl der erlaubten Übergänge kann noch wesentlich größer sein. Die Intensität des Gesamtspektrums, umgerechnet auf ein Molekül, ist aber nur proportional der Protonenzahl. Die große Anzahl der Linien ist also verbunden mit einer sehr geringen Intensität der Einzellinien, so daß es schon große Schwierigkeiten bereitet, auch nur einen Teil der Feinstruktur des Spektrums zu erhalten.

14 Protonen (wie bei Azoxyanisol) ist für eine kristallinflüssige Substanz eine verhältnismäßig geringe Anzahl. Die Kernresonanzspektren vieler kristalliner Flüssigkeiten (höhere Homologe) sind da- her im allgemeinen noch viel linienreicher und die Intensität der Einzellinie noch viel geringer. Es ist deshalb möglich, Kernresonanzspektren von gelösten Molekülen aufzunehmen, auch wenn ihre Resonanzlinien im Absorptionsgebiet der kristallinflüssigen Trägersubstanz liegen.

Löst man Moleküle, die im Vergleich zur kristallinflüssigen Trägersubstanz nur eine kleine Anzahl von wechselwirkenden Kernen enthalten, so liefern die gelösten Moleküle relativ wenige, aber trotz geringerer Konzentration starke Signale, die sich deutlich aus dem Spektrum der Trägersubstanz herausheben. Sie können in günstigen Fällen in Hochauflösung nach dem stationären Verfahren beobachtet werden, wo die kristallinflüssige Trägersubstanz nur ein breites Untergrundsignal liefert. Man erhält auf diese Weise also hochaufgelöste Spektren von orientierten Molekülen. Wir haben schon eine Anzahl von Substanzen in nematischen Lösungen untersucht und interessante Ergebnisse erhalten, über die wir in folgenden Arbeiten berichten werden.

Herrn Prof. Dr. W. MaIER danke ich für viele Anregungen, sein Interesse an dieser Arbeit und für die tatkräftige Förderung; Herrn Prof. Dr. R. Mecke danke ich für seine entgegenkommende Unterstützung; Herrn Dr. G. ENGLERT, mit dem ich eng zusammenarbeitete, bin ich verbunden für klärende Diskussionen und die Überlassung der Spektren.

Dem Bundesministerium für wissenschaftliche Forschung danke ich für ein Forschungsstipendium, das mir diese Arbeit ermöglicht hat, der Deutschen Forschungsgemeinschaft und dem Fonds der Chemie für ihre Förderung unserer Untersuchungen über kristallinflüssige Phasen. 